\title{
Nanostructures from Molecules
}

\begin{abstract}
Recent successes in creating structures from individual atoms and molecules at temperatures appreciably above $4 \mathrm{~K}$ give rise to the hope that they will be built and used routinely to investigate fundamental phenomena.
\end{abstract}

The vision of scientists and engineers to build new structures and maybe even new materials atom-by-atom is certainly as old as mankind's knowledge of the existence of atoms. The first important step towards realizing this dream was made by D.M. Eigler and collaborators [1] at the IBM-Almaden Research Laboratory employing the scanning tunnelling microscope (STM) [2]. The quantum laboratories of Eigler et al. [3] made certain phenomena literally visible, notable those which concern the most abstract foundations of quantum mechanics [4]. The fact that we have recently succeeded [5] in build ing artificial structures at temperatures appreciably above the temperature $(4 \mathrm{~K})$ at which the previous experiments were performed gives rise to the hope that, with the STM, man-made patterns can be built routinely in the future from atoms and molecules.

As an example of our manipulations on the atomic scale we show in Fig. 1 the letters " $F$ " and "U" which form the logo of our university. Both structures were built at $30 \mathrm{~K}$ using our variable temperature, low-temperature STM operating in an ultra-high vacuum. We used $\mathrm{CO}$ molecules as the building blocks and a $\mathrm{Cu}$ (211) single crystal surface as the substrate. The CO molecules are imaged as dark spots. The face-centred cubic (211) surfaces consist of (111) nanofacets and steps with a (100) orientation. The straight bright lines in Fig. 1 correspond to the uppermost $\mathrm{Cu}$ atoms located at the step edges. The CO molecules adsorb at the step edges with the carbon atoms pointing towards the metal atoms.

We used a procedure analogous to that of Eigler et al. [1] to manipulate the CO molecules. In the STM imaging mode the tip is scanned at distances of a few atomic diameters above the surface and the image corresponds to contours of constant local electronic density of states if the STM is regulated to operate at constant current. To manipulate a single adsorbed species the tip is brought close to a given adsorbate by reducing the tunnelling resistance. It is then moved parallel to the surface by a given distance whereupon the atom or molecule is dragged to the desired place. Finally, the tip is withdrawn to a larger distance and the result of the manipulation checked by scanning to obtain a new STM image. Precise lateral movements of single $\mathrm{CO}$ molecules parallel to the $\mathrm{Cu}$ (211) step edges were obtained by reducing the tunnelling resistance to a few megaohms.

Fig. 2 illustrates, using as an example the formation of the letter " $F$ ", how an area of reduced adsorbate density must first be produced out of the initial statistical distribution of $\mathrm{CO}$ molecules; the nanostructure is then built up from the required number of adsorbed particles. The first STM image in the series corresponds to a situation in which many molecules have already been removed from the central area. The thermal mobility of the CO molecules at $30 \mathrm{~K}$ is still large enough for irregular jumps to disturb the order (thermal jumps also cause the charming irregularities of the two letters shown in Fig. 1). Owing to the pronounced anisotopy of the Cu (211) substrate, manipulation of the $\mathrm{CO}$ molecules along the step edges proceeds readily whereas movements perpendicular to the steps are difficult. We also point out that whereas every step edge is initially occupied by $\mathrm{CO}$ molecules, in the areas used to form the nanoletters all molecules were removed from every second step.

Using our STM we can vary the sample temperature between $15 \mathrm{~K}$ and room temperature so new experiments of interest for both physics and chemistry become possible. Investigations of diffusion and the thermal stability of nanostructures are as attractive as both manipulation of different species coadsorbed at surfaces and structure formation
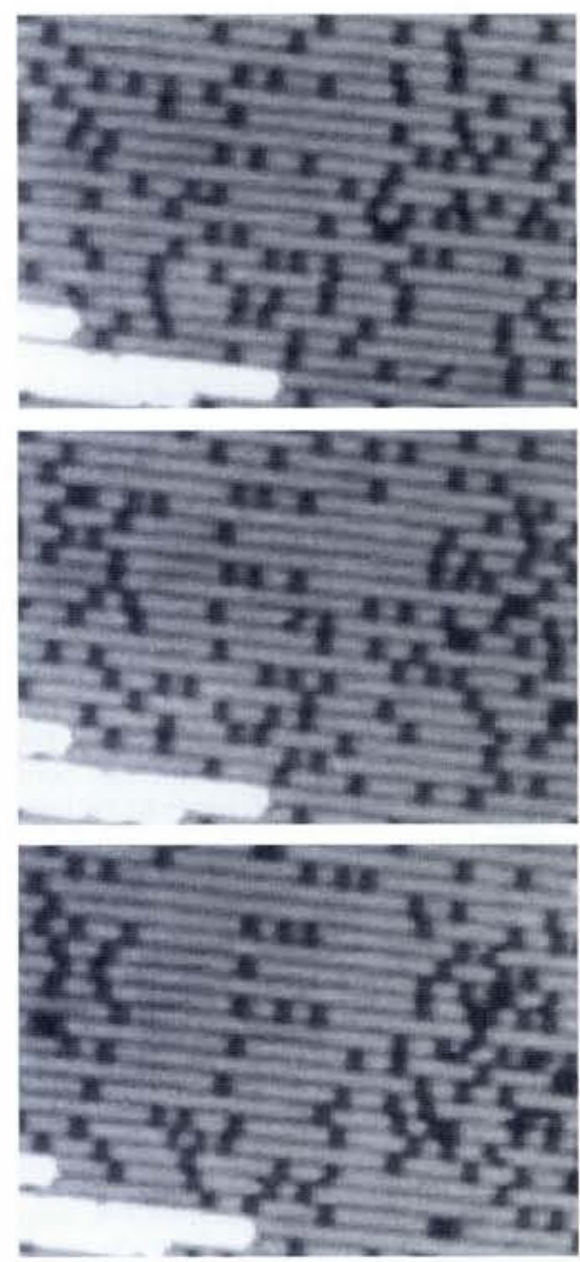

Fig. $2-A$ sequence of STM images showing the final phase of the build-up of the letter " $F$ " from an area of reduced adsorbate density. The horizontal size of each image is $16 \mathrm{~nm}$. Same experimental conditions as for Fig. 1 .

using more than one sort of adsorbed particle. Each requires investigation of the possibility of manipulating different species, especially atoms of metals and semiconductors where the degree of substrate corrugation and the ability to pull adsorbed species in different directions need special attention (and should also interest theorists). Since we can also transfer single $\mathrm{Xe}$ atoms at $15 \mathrm{~K}$ to the tip, the role of the chemical composition of the tip in the imaging process itself can be studied. Furthermore, the construction of three-dimensional nanostructures appears feasible because adatoms at the tip can be transported to some desired place and released back to the surface $[1,6]$.

Fig. 1 - Scanning pe images of the letters " $F$ " and " $U$ " (short for the Free University, Berlin) built up with $\mathrm{CO}$ molecules on a $\mathrm{Cu}$ (211) substrate by controlled lateral manipulation of the molecules with the microscope tip. The substrate tempera-
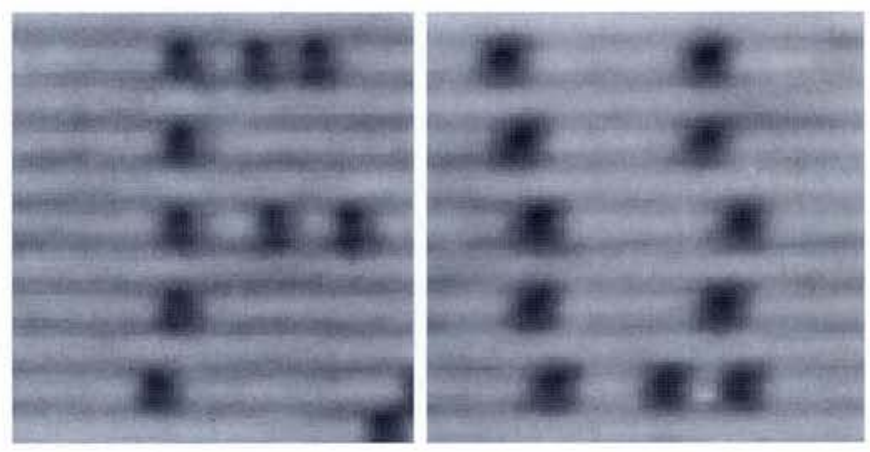

ture was $30 \mathrm{~K}$ and the vertical size of the images corresponds to $6 \mathrm{~nm}$. The experimental conditions were: $0.2 \mathrm{~V}$ applied to the sample; $100 \mathrm{pA}$ tunnelling current for imaging and 100$200 \mathrm{nA}$ for manipulation; $5 \mathrm{~nm} / \mathrm{sec}$ dragging velocity.

[1] Eigler D.M. \& Schweitzer E.K., Nature 344 (1990) 524; Stroscio J.A. \& Eigler D.M., Science 254 (1991) 1319; Zeppenfeld P., Lutz C.P. \& Eigler D.M., Uitramicroscopy 42-44 (1992) 128.

[2] Binnig G. \& Rohrer H., Rev. Mod. Phys. 59 (1987) 615.

[3] Crommie M.F., Lutz C.P. \& Eigler D.M., Nature 262 (1993) 218; Heller E.J. et al., Nature 369 (1994) 464.

[4] Hörmandinger G., Europhys. News 25 (1994) 148.

[5] Meyer G., Neu B. \& Rieder K.H., Appl. Phys. A 60 (March 1995); to be published.

[6] Lyo L.W. \& Avouris P., Science 253 (1991) 173.

G. Meyer, B. Neu, K.H. Rieder Freie Universität Berlin 